\title{
IMPLICAÇÕES CLÍNICAS E PRODUTIVAS DO PH URINÁRIO DE VACAS LEITEIRAS DE ALTA PRODUÇÃO NO PERÍODO PRÉ-PARTO
}

\author{
Tiago André Frigotto ${ }^{1}$, Rodrigo Barros Navarro ${ }^{2}$, Patrick Schmidt ${ }^{1}$, Ivan Roque de \\ Barros Filho ${ }^{1}$, Rüdiger Daniel Ollhoff ${ }^{3}$, Rodrigo de Almeida ${ }^{1}$ \\ 1 Universidade Federal do Paraná - ralmeida@ufpr.br \\ 2 Capal Cooperativa Agroindustrial \\ 3 Pontifícia Universidade Católica do Paraná
}

RESUMO: O estudo teve como objetivo correlacionar dados de $\mathrm{pH}$ urinário no pré-parto de vacas leiteiras de alta produção com níveis sanguíneos de cálcio $(\mathrm{Ca})$, incidência de doenças metabólicas nos primeiros 10 dias de lactação, ordem de lactação e produção de leite no pós-parto. Cento e cinco vacas leiteiras pertencentes a dois rebanhos comerciais foram suplementadas nos últimos 21 dias de gestação com sal aniônico. A diferença catiônica-aniônica da dieta (DCAD) foi estimada em $+70 \mathrm{mEq} / \mathrm{kg}$ de matéria seca (MS). Amostras de sangue foram colhidas nos dias 1, 2, 5 e 10 após o parto para determinação da concentração de Ca total. Durante os primeiros 10 dias de lactação realizou-se o diagnóstico de doenças metabólicas, por meio de exame clínico, e mediu-se a produção de leite diariamente. $\mathrm{O}$ valor médio do $\mathrm{pH}$ urinário pré-parto foi de $8,2 \pm 0,4$. Níveis médios de Ca sanguíneo nos dias 1, 2, 5 e 10 foram de 10,29, 10,32, 11,07 e 11,22 mg/dL, respectivamente. Das 105 vacas monitoradas, 44,8\% apresentaram alguma enfermidade durante os primeiros 10 dias após o parto. Não foram observadas correlações significativas $(P>0,05)$ entre o $\mathrm{pH}$ urinário pré-parto com os níveis de Ca sérico e a incidência de doenças metabólicas.

Palavras-chave: bovinocultura leiteira; diferença catiônica-aniônica da dieta; doenças metabólicas; período de transição

\section{CLINICAL AND PRODUCTIVE IMPLICATIONS OF URINARY PH OF HIGH- PRODUCING DAIRY COWS DURING THE PREPARTUM PERIOD}

\begin{abstract}
The objective of this trial was to correlate prepartum urinary $\mathrm{pH}$ data of high-producing dairy cows with blood calcium levels, metabolic diseases incidence in the first ten days of lactation, lactation number and milk yield in the postpartum. One hundred and five dairy cows belonging to two commercial herds were supplemented with anionic salt in the last 21 days of pregnancy. The dietary cationic-anionic difference (DCAD) was estimated at $+70 \mathrm{mEg} / \mathrm{kg}$ of dry matter. Blood samples were collected on days $1,2,5$ and 10 after calving to evaluate total calcium $(\mathrm{Ca})$ concentration. During the first 10 days of lactation, individual metabolic diseases and daily milk yield were recorded. Average prepartum urinary $\mathrm{pH}$ was $8.2 \pm 0.4$. Average serum $\mathrm{Ca}$ on days $1,2,5$ and 10 were 10.29, 10.32, 11.07 and $11.22 \mathrm{mg} / \mathrm{dL}$, respectively. From the 105 monitored cows, $44.8 \%$ showed at least one disease event in the first 10 days postpartum. There were no significant correlations $(P>0.05)$ between prepartum urinary $\mathrm{pH}$ and blood $\mathrm{Ca}$ levels, as well as no significant associations $(P>0.05)$ between prepartum urinary $\mathrm{pH}$ and the metabolic diseases incidence.
\end{abstract}

Key Words: dairy production; dietary cation-anion difference; metabolic diseases; transition period 


\section{INTRODUÇÃO}

A grande exigência de cálcio ( $\mathrm{Ca})$ sanguíneo pela glândula mamária para produção do colostro, associado a um temporário desequilíbrio entre ingestão e exigência ao parto, resulta em baixos níveis séricos deste mineral no período periparto. A baixa concentração de $\mathrm{Ca}$ promove a ocorrência de doenças metabólicas, principalmente hipocalcemia, particularmente em vacas leiteiras multíparas e de alta produção (Horst et al., 1997; Seifi et al., 2004; Wu et al., 2008).

A súbita demanda de $\mathrm{Ca}$ no início da lactação é um severo teste para a capacidade de homeostasia do $\mathrm{Ca}$ no animal (Goff, 2008). A manutenção da homeostasia do Ca é essencial para muitas funções, incluindo a excitabilidade neuromuscular, coagulação sanguínea e secreção de hormônios (Wu et al., 2008).

Baixos níveis de $\mathrm{Ca}$ sanguíneo causam redução na ingestão de alimentos, menor motilidade ruminal e intestinal, decréscimo no volume de leite produzido e aumento na suscetibilidade de outras doenças metabólicas e infecciosas (Goff, 2008). Degaris e Lean (2008) citaram que vacas com hipocalcemia possuem um alto risco de serem acometidas por outras importantes enfermidades, incluindo cetose, mastite, retenção de placenta, deslocamento de abomaso e prolapso uterino.

A hipocalcemia continua sendo uma das doenças de maior importância econômica para a bovinocultura leiteira (Seifi et al., 2004). De acordo com Hutjens e Aalseth (2005), a perda causada por esta enfermidade, para cada vaca acometida, chega a $R \$$ 575,00 (assumindo cotação do dólar em $\mathrm{R} \$ 1,72)$. Sendo assim, medidas para prevenção desta doença devem ser tomadas. Block (1984) citou diferentes formas de prevenção, entretanto, o mé- todo mais eficiente é o aumento da inclusão de minerais acidogênicos na dieta de vacas leiteiras nas três semanas que antecedem o parto, bem como evitar o fornecimento de forragens ricas em potássio, ou seja, ajustar a diferença catiônica-aniônica da dieta (DCAD) (NRC, 2001; Beauchemin et al., 2003).

A deficiência de $\mathrm{Ca}$ no início da lactação ativa mecanismos fisiológicos com o objetivo de suprir este déficit, retirando $\mathrm{Ca}$ dos ossos e aumentando a eficiência de absorção de Ca oriundo da dieta. Esta regulação é feita pelo paratormônio (PTH), secretado pela paratireóide, que detecta a redução da concentração de $\mathrm{Ca}$ sanguíneo estimulando a reabsorção óssea de $\mathrm{Ca}$ pelos osteoclastos e estimulando a produção renal de 1,25-dihidroxivitamina $D_{3}$, que estimula as células epiteliais do intestino a aumentar a absorção do $\mathrm{Ca}$ dietético. Uma alcalose metabólica no organismo diminui a ação do PTH, impedindo a ativação da reabsorção óssea. Por este motivo preconiza-se a formulação de dietas aniônicas no préparto, para que provoquem uma moderada acidose metabólica e com isso, potencializem a ação do PTH (Goff, 2009).

Dietas aniônicas diminuem 0 potencial hidrogeniônico $(\mathrm{pH})$ sanguíneo e consequentemente $\mathrm{o} \mathrm{pH}$ urinário. Sendo assim, o monitoramento do $\mathrm{pH}$ urinário é um método eficiente para determinação da resposta do animal frente a dieta aniônica fornecida. Leite et al. (2003) demonstraram que esta metodologia é eficiente na avaliação da funcionalidade da acidificação da dieta aniônica e por consequência na prevenção de doenças metabólicas em vacas periparturientes (Seifi et al., 2004).

O objetivo deste trabalho foi de correlacionar dados de $\mathrm{pH}$ urinário no 
pré-parto de vacas leiteiras de alta produção com níveis sanguíneos de $\mathrm{Ca}$, incidência de doenças metabólicas nos primeiros 10 dias de lactação, ordem de lactação e produção de leite no pósparto.

\section{MATERIAL E MÉTODOS}

Dois rebanhos leiteiros comerciais no município de Arapoti, estado do Paraná, foram monitorados de 01 de abril a 12 de julho de 2009. Ambos os rebanhos possuíam o mesmo sistema intensivo de criação do tipo "free-stall", com ordenha realizada três vezes ao dia e média de produção de 35 litros de leite/vaca/dia. As dietas fornecidas foram balanceadas de acordo com as exigências nutricionais para vacas no período de transição (NRC, 2001, Tabelas 1 e 2). Adicionalmente foi incluído na dieta total, um suplemento mineral aniônico pré-parto, na quantidade de 150 gramas divididos em dois tratos diários. Após pesado, o sal aniônico era manualmente misturado à dieta total. Esta dieta foi fornecida nos últimos 21 dias da data prevista para 0 parto.

Neste trabalho a DCAD foi calculada usando a fórmula sugerida por Hutjens e Aalseth (2005): DCAD $=(\% \mathrm{Na}$ x $434+\% \mathrm{~K} \times 256)-(\% \mathrm{Cl} \times 282+\% \mathrm{~S} \times$ 624). A composição mineral da dieta pré-parto em porcentagem da MS era $0,20 \% \mathrm{Na}, 1,65 \% \mathrm{~K}, 0,76 \% \mathrm{Cl}$ e $0,36 \% \mathrm{~S}$. A partir destes valores, estimou-se a DCAD da dieta em $+70 \mathrm{mEq} / \mathrm{kg}$ de MS.

Avaliou-se um total de 105 vacas da raça Holandesa, sendo 26 (24,8\%) da propriedade A e $79(75,2 \%)$ da propriedade $B$, das quais 73 eram multíparas $(69,5 \%)$ e 32 primíparas $(30,5 \%)$. Do total de 105 vacas, 58 $(55,2 \%)$ foram classificadas como sadias e 47 (44,8\%) apresentaram algum distúrbio clínico após o parto (retenção de placenta, edema de úbere, deslocamento de abomaso, endometrite puerperal, mastite, broncopneumonia e hipocalcemia clínica).

Para 0 diagnóstico das enfermidades foi realizado diariamente, após a ordenha da manhã, em todas as vacas recém-paridas, do primeiro ao décimo dia, o exame clínico nos animais. Avaliou-se o sistema digestório através de ausculta ruminal e percussão auscultatória do abdômen conforme metodologia descrita por Dirksen (1993), além da aferição da temperatura retal e urinálise por meio de fitas reagentes a base de nitroprussinato de sódio para verificação de corpos cetônicos.

A urina foi colhida no período da manhã em recipientes de $50 \mathrm{~mL}$, durante a micção espontânea ou pela realização de massagem perineal. Logo após a colheita, o pH das amostras foi medido por meio de peagametro portátil da marca Quimis (modelo Q400B). A colheita foi realizada entre os últimos sete dias de gestação de acordo com a data prevista para o parto.

Coletaram-se amostras de sangue para análise de cálcio total, após a ordenha da manhã e antes da primeira refeição diária, nos dias 1, 2, 5 e 10 pósparto, mediante punção venosa coccígea em tubos sem anticoagulante. Após a colheita as amostras de sangue foram transportadas em temperatura ambiente para o Laboratório de Análises Clínicas - BIOLAB, na cidade de Arapoti, Paraná, sendo centrifugadas a 3000 rpm por 10 minutos para obtenção de soro, que em seguida era aspirado e dividido em duas frações armazenadas em tubos plásticos de 1,5 $\mathrm{mL}$, identificadas e congeladas a $-20^{\circ} \mathrm{C}$ até a realização das análises.

Os teores de $\mathrm{Ca}$ sérico foram quantificados por metodologia enzimática colorimétrica em analisador bioquímico automático da marca Biosystems, modelo A15, utilizando-se kits reagentes comerciais da marca Randox®. Os resultados foram expressos em mg/dL. 
Tabela 1 - Composição da dieta oferecida às vacas no período pré-parto

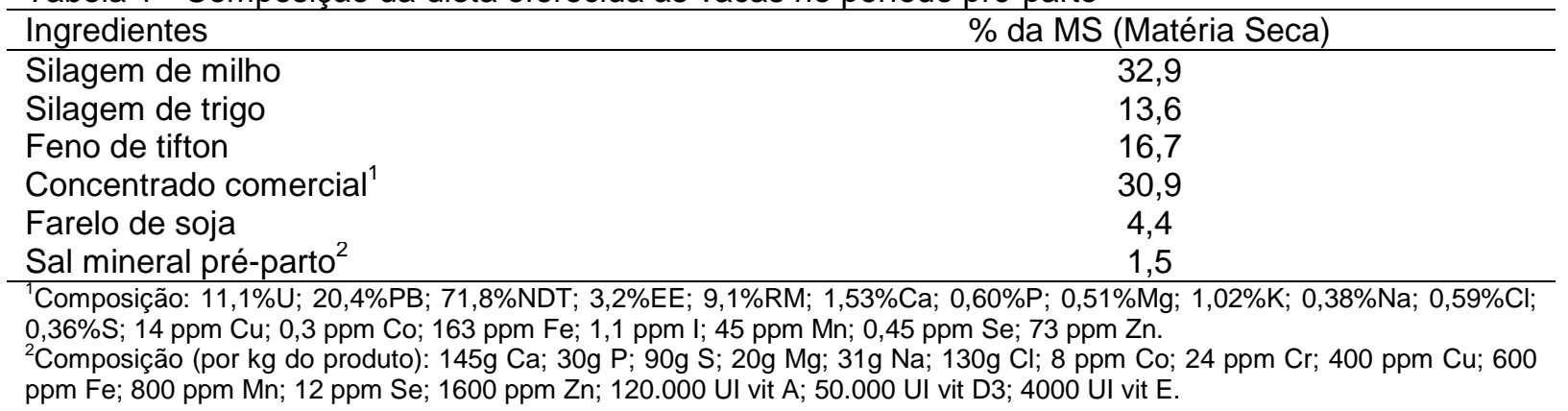

Tabela 2 - Composição química das forragens oferecidas às vacas no período pré-parto

\begin{tabular}{|c|c|c|c|}
\hline Nutriente (\%MS) & Silagem de milho & Silagem de trigo & Feno de tifton \\
\hline Matéria Seca (MS) & 33,2 & 34,1 & 84,9 \\
\hline Proteína Bruta (PB) & 8,10 & 10,17 & 13,66 \\
\hline $\mathrm{NDT}^{1}$ & 71,30 & 59,94 & 48,60 \\
\hline Fibra Detergente Neutra (FDN) & 38,39 & 61,95 & 80,51 \\
\hline Fibra Detergente Ácida (FDA) & 23,46 & 37,47 & 41,44 \\
\hline $\mathrm{CNF}^{2}$ & 45,82 & 20,17 & \\
\hline Extrato Etéreo (EE) & 4,32 & 2,47 & 1,81 \\
\hline Resíduo Mineral (RM) & 3,37 & 5,24 & 7,78 \\
\hline Cálcio (Ca) & 0,13 & 0,28 & 0,54 \\
\hline Fósforo $(P)$ & 0,22 & 0,20 & 0,20 \\
\hline
\end{tabular}

${ }^{\mathrm{l}}$ Nutrientes Digestíveis Totais, estimado usando a equação de Weiss (NRC, 2001).

${ }^{2}$ Carboidratos não-fibrosos $=100-($ PB + FDN + EE + RM).

A análise estatística foi realizada pelo procedimento GLM do SAS (2002) e no modelo matemático foram incluídos os efeitos fixos de fazenda (A e B), de ordem de parto (primíparas e multíparas), natureza do parto (simples e gemelar) e ocorrência de enfermidades (sadias e doentes), além da inclusão da covariável produção de leite ajustada para o $10^{\circ}$ dia pós-parto. As médias ajustadas para as diferentes variáveis foram comparadas ao nível de $1 \% \quad(P<0,01) \quad$ e $5 \% \quad(P<0,05) \quad$ de probabilidade.

\section{RESULTADOS E DISCUSSÃO}

Os pH's urinários no pré-parto das vacas são apresentados na escala sugerida por Jardon (1995): menor que 6 , entre 6 e 7, 7 e 8, e maior que 8 . 0 número de animais, a porcentagem correspondente a cada intervalo de $\mathrm{pH}$ e $0 \mathrm{pH}$ médio de cada classe podem ser observados na Tabela 3.
Segundo Jardon (1995), Oetzel (2004), Hutjens e Aalseth (2005) e Goff (2009), os valores ideais de $\mathrm{pH}$ urinário na semana que antecede o novo parto devem estar entre 6,0 e 7,0. Já que poucos animais deste estudo atingiram o pH urinário desejável, pode-se afirmar que a dieta fornecida não era de fato aniônica e, portanto, não promoveu a desejável acidose metabólica. Isto deve ter ocorrido ou pela pequena inclusão do sal pré-parto, ou pela inclusão de forragens com alto teor de potássio, ou ainda, mais provavelmente, pela somatória dos dois fatores.

O período de fornecimento do sal aniônico, nos últimos 21 dias da data prevista para o parto, foi considerado suficiente por ser o mesmo adotado em outros trabalhos (Horst et al., 1997, Leite et al., 2003, Hu et al., 2007, Wu et al., 2008, Ramos-Nieves et al., 2009), inclusive numa meta-análise que reuniu 22 trabalhos contendo 75 distintos tratamentos (Charbonneau et al., 2006). 
Tabela 3 - Distribuição dos animais por classe de pH urinário no pré-parto

\begin{tabular}{|c|c|c|c|}
\hline Escala de $\mathrm{pH}$ & No. animais & Porcentagem & pH médio \\
\hline$<6$ & 1 & 0,95 & 5,60 \\
\hline 6 a 7 & 1 & 0,95 & 6,30 \\
\hline 7 a 8 & 26 & 24,76 & $7,89 \pm 0,13$ \\
\hline$>8$ & 77 & 73,33 & $8,39 \pm 0,18$ \\
\hline Total & 105 & 100 & $8,22 \pm 0,42$ \\
\hline
\end{tabular}

Observou-se um valor médio de $\mathrm{pH}$ urinário de 8,22 \pm 0,42 entre os 105 animais avaliados. O mesmo valor foi observado por Ramos-Nieves et al. (2009), fornecendo dieta com DCAD de $+110 \mathrm{mEq} / \mathrm{kg}$ de MS. Leite et al. (2003) observaram valor próximo de $\mathrm{pH}(7,9)$ fornecendo dieta com DCAD de +122 $\mathrm{mEq} / \mathrm{kg}$ de MS. Dietas com DCAD positivo promovem alcalose metabólica; tal fato é evidenciado no $\mathrm{pH}$ urinário que torna-se alcalino, como pode ser observado neste e nos demais trabalhos citados acima.

Alcalose metabólica é o resultado de uma dieta que fornece mais cátions (K, $\mathrm{Na}, \mathrm{Ca}$ e $\mathrm{Mg}$ ) que ânions (cloretos, sulfatos e fosfatos) ao sangue. Esta alcalose predispõe à ocorrência de doenças metabólicas no pós-parto, entre as quais a hipocalcemia subclínica e clínica, por diminuir a mobilização óssea e a absorção intestinal de $\mathrm{Ca}$, fazendo com que os níveis deste mineral durante o periparto permaneçam baixos (Goff, 2009; Degaris e Lean, 2008).

A hipocalcemia pode apresentarse nas formas subclínica, a qual não apresenta sinais clínicos, e clínica, que demonstra sinais clínicos da doença. Para um correto diagnóstico e classificação destas formas, a análise sérica torna-se uma importante ferramenta. Níveis considerados normais estão acima de $7,5 \mathrm{mg} / \mathrm{dL}$. Abaixo deste, até $5 \mathrm{mg} / \mathrm{dL}$, considera-se como hipocalcemia subclínica, e níveis menores que $5 \mathrm{mg} / \mathrm{dL}$, há ocorrência de uma hipocalcemia clínica (Goff, 2009). A distribuição de vacas em cada um des- tes grupos no presente experimento pode ser visualizada na Tabela 4.

Os níveis médios de $\mathrm{Ca}$ sérico observados neste trabalho foram, nos dias 1, 2, 5 e 10 pós-parto de 10,29, $10,32, \quad 11,07$ e 11,22 mg/dL, respectivamente (Tabela 4). A partir destes valores, pode-se afirmar que nos dois rebanhos avaliados, não houve deficiência de Ca nos primeiros 10 dias após o parto, ainda que a DCAD préparto foi de $+70 \mathrm{mEq} / \mathrm{kg} \mathrm{MS}$. O fato de que uma dieta com balanço cationicoaniônico negativo promove uma acidose metabólica e por consequência um aumento na concentração sérica de $\mathrm{Ca}$, conforme relatado por diversas pesquisas (Charbonneau et al., 2006, Wu et al., 2008, Ramos-Nieves et al., 2009), não pôde ser demonstrado neste trabalho pelo fato da DCAD ser positiva.

Das 105 vacas monitoradas, $44,8 \%$ apresentaram alguma enfermidade durante os primeiros 10 dias após o parto; 11 vacas apresentaram distocia, 27 retenção de placenta, 21 edema puerperal e 7 torção de abomaso. Esta incidência de 44,8\% é similar à relatada por Drackley (1999), em rebanhos norte-americanos de alta produção, onde aproximadamente $50 \%$ das vacas recém-paridas apresentaram ao menos uma anormalidade no pósparto.

No entanto, as incidências de hipocalcemia subclínica (níveis sanguíneos de $\mathrm{Ca}$ menores que 7,5 $\mathrm{mg} / \mathrm{dL}$ ) e clínica (inferiores a 5,0 mg/dL) no dia seguinte ao parto, 10,5 e $0,95 \%$, respectivamente, foram baixas e consideradas aceitáveis por Oetzel (2004), que sugere níveis alarmantes a 
Tabela 4 - Níveis médios de cálcio sérico e número de vacas apresentando calcemia adequada, hipocalcemia subclínica e hipocalcemia clínica

\begin{tabular}{lcccc}
\hline \multicolumn{1}{c}{ Dias pós-parto } & Média \pm DP & $\begin{array}{c}\text { No. vacas com } \\
\text { calcemia } \\
\text { adequada }^{1}\end{array}$ & $\begin{array}{c}\text { No. vacas com } \\
\text { hipocalcemia } \\
\text { subclínica }^{2}\end{array}$ & $\begin{array}{c}\text { No. vacas com } \\
\text { hipocalcemia }^{\text {clínica }^{3}}\end{array}$ \\
\hline Dia1 & $10,29 \pm 3,37$ & 93 & 11 & 1 \\
Dia 2 & $10,32 \pm 2,93$ & 100 & 5 & 0 \\
Dia 5 & $11,07 \pm 2,80$ & 105 & 0 & 0 \\
Dia 10 & $11,22 \pm 2,47$ & 105 & 0 & 0 \\
\hline
\end{tabular}

${ }^{1}$ Níveis de cálcio sérico acima de 7,5 mg/dL.

${ }^{2}$ Níveis de cálcio sérico entre 5,0 e 7,5 mg/dL.

${ }^{3}$ Níveis de cálcio sérico abaixo de $5,0 \mathrm{mg} / \mathrm{dL}$.

partir de 30 e $8 \%$ para hipocalcemia subclínica e clínica, respectivamente. De maneira oposta, observaram-se altas incidências de retenção de placenta $(25,7 \%)$ e de deslocamento de abomaso $(6,7 \%)$, sendo que os limites aceitáveis sugeridos por Overton et al. (2002) para estas desordens são taxas inferiores a $8 \%$ e $3 \%$, respectivamente.

Não foram observadas diferenças $(\mathrm{P}>0,05)$ de $\mathrm{pH}$ urinário das vacas no pré-parto entre as duas fazendas participantes, entre vacas de parto gemelar e simples, e entre vacas sadias e doentes. Wu et al. (2008), comparando dietas pré-parto com DCAD positivas contra negativas, observaram que vacas recebendo dietas com DCAD de $+150 \mathrm{mEq} / \mathrm{kg}$ MS obtiveram média de $\mathrm{pH}$ urinário de 7,67 , e que neste grupo, $40 \%$ das vacas apresentaram retenção de placenta. Já no grupo que recebeu dieta com DCAD de $-150 \mathrm{mEq} / \mathrm{kg} \mathrm{MS}$, observou-se média de $\mathrm{pH}$ urinário de 5,75, sem a ocorrência de casos de retenção de placenta. Este fato demonstra que a acidose metabólica promovida pela DCAD negativa e por consequência o acréscimo nos níveis de $\mathrm{Ca}$ sérico, aumentou o tônus uterino, melhorou a motilidade muscular e incrementou a resposta imune, evitando a retenção dos anexos fetais no pós-parto.

A incidência de hipocalcemia subclínica não foi correlacionada com o pH urinário ácido, de acordo com Ramos-Nieves et al. (2009). Da mesma forma, Wu et al. (2008) também não observaram associação entre $\mathrm{pH}$ urinário ácido com as incidências de hipocalcemia clínica e subclínica, edema de úbere, deslocamento de abomaso e mastite.

Com relação ao efeito de ordem de parição, este mostrou-se importante fonte de variação $(P<0,05)$, onde vacas primíparas apresentaram $\mathrm{pH}$ urinário no pré-parto superior ao de vacas multíparas $(8,38$ versus 8,12$)$. Por fim, a inclusão da covariável produção de leite corrigida para $010^{\circ}$ dia pós-parto também apresentou significância $(\mathrm{P}<0,01)$.

Correlações simples de Pearson foram estimadas entre o pH urinário préparto e diversas variáveis analisadas (Tabela 5).

Não foram encontradas correlações significativas $(P>0,05)$ entre o $\mathrm{pH}$ urinário pré-parto e as análises de Ca total nos dias 1, 2, 5 e 10. Este resultado pode ser justificado pela DCAD ser positiva, a qual não promoveu um aumento nos níveis séricos de $\mathrm{Ca}$. Charbonneau et al. (2006); Wu et al. (2008) e RamosNieves et al. (2009) demonstraram que dietas pré-parto, quando aniônicas, são eficientes no aumento de $\mathrm{Ca}$ total no pós-parto. Entretanto, Roche et al. (2007) não observaram este aumento de $\mathrm{Ca}$ ao fornecer uma dieta com DCAD negativa.

A correlação entre $\circ \mathrm{pH}$ urinário pré-parto e a produção de leite no pósparto foi significativa $(P<0,05)$ e positiva $(r=+0,24)$, indicando que as vacas 
Tabela 5 - Correlações fenotípicas entre o pH urinário no pré-parto e algumas variáveis

\begin{tabular}{lcc}
\multicolumn{1}{c}{ Variáveis } & $\mathrm{r}$ & $P$ \\
\hline Idade (meses) & $-0,17$ & 0,07 \\
Ca no dia 1pp (mg/dL) & $+0,13$ & 0,20 \\
Ca no dia 2pp (mg/dL) & $+0,04$ & 0,66 \\
Ca no dia 5pp (mg/dL) & $+0,03$ & 0,76 \\
Ca no dia 10pp (mg/dL) & $+0,11$ & 0,25 \\
PLCD10 & $+0,24$ & 0,02 \\
\hline
\end{tabular}

Produção de leite corrigida para o 10 dia pós-parto.

com maior $\mathrm{pH}$ urinário pré-parto produziram mais leite no pós-parto. Por outro lado, Hu et al. (2007); Wu et al. (2008) e Ramos-Nieves et al. (2009) não observaram diferenças significativas na produção de leite de vacas com $\mathrm{pH}$ urinário ácido com relação às que apresentaram $\mathrm{pH}$ básico. Roche et al. (2003) avaliaram quatro dietas com diferentes DCAD positivas, também não encontrando diferenças significativas na produção de leite.

\section{CONCLUSÃO}

A adição de um sal aniônico na dieta pré-parto não garante a ocorrência de uma DCAD negativa e consequentemente uma leve acidose metabólica. Mais importante que adicionar um sal pré-parto é verificar a DCAD da dieta e principalmente, evitar que volumosos com altos teores de potássio sejam fornecidos às vacas nas últimas semanas que antecedem 0 parto.

Quando a DCAD é positiva e a desejável acidose metabólica não é alcançada, não há correlações significativas entre $\mathrm{pH}$ urinário no préparto com níveis de $\mathrm{Ca}$ sérico e ocorrência de desordens metabólicas no pós-parto.

\section{AGRADECIMENTOS}

Ao corpo técnico da Química Geral do Nordeste (QGN) e Laboratórios Pfizer Ltda. pelo auxílio financeiro que viabilizou a aquisição dos kits comerciais. Ao laboratório de análises clínicas Biolab de Arapoti, por disponibilizar o equipamento para realização das análises séricas. Aos Médicos Veterinários Jan Willem e Marika Salomons, proprietários da Fazenda D’Água Verde e ao Zootecnista Adriaan Kok, proprietário da Chácara Baronesa.

\section{REFERÊNCIAS}

BEAUCHEMIN, K. A.; BOWMAN, G. R.; RODE, L. M. et al. Effects of feeding anionic products to non-lactating dairy cows on urine $\mathrm{pH}$. Canadian Journal of Animal Science, v.83, n.3, p.609612, 2003.

BLOCK, E. Manipulating dietary anions and cations for prepartum dairy cows to reduce incidence of milk fever. Journal of Dairy Science, v.67, n.12, p.2939-2948, 1984.

CHARBONNEAU, E.; PELLERIN, D.; OETZEL, G. R. Impact of lowering dietary cation-anion difference in nonlactating dairy cows: a metaanalysis. Journal of Dairy Science, v.89, n.2, p.537-548, 2006.

DEGARIS, P. J.; LEAN, I. J. Milk fever in dairy cows: A review of pathophysiology and control principles. The Veterinary Journal, v.176, n.1, p.58-69, 2008.

DIRKSEN, G. Sistema digestório. In: DIRKSEN, G.; GRÜNDER, H.D.; STÖBER, M. Rosenberger - Exame clínico dos bovinos. 3.ed. Rio de Janeiro: Guanabara Koogan, 1993, Cap.7, p.166-228.

DRACKLEY, J.K. Biology of dairy cows during the transition period: the final frontier? Journal of Dairy Science, v.82, n.11, p.2259-2273, 1999.

GOFF, J. P. Como controlar a febre do leite e outras desordens metabólicas relacionadas a macro minerais em vacas de leite. In: XIII CURSO DE NOVOS ENFOQUES NA PRODUÇÃO E REPRODUÇÃO DE BOVINOS., 2009, Uberlândia. Anais... Botucatu: Universidade Estadual Paulista, 2009, p. 267284. CD-ROM

GOFF, J. P. The monitoring, prevention, and treatment of milk fever and subclinical hypocalcemia in dairy cows. The Veterinary Journal, v.176, n.1, p.50-57, 2008.

HORST, R.L.; GOFF, J.P.; REINHARDT, T.A. et al. Strategies for preventing milk fever in dairy 
cattle. Journal of Dairy Science, v.80, n.7, p.1269-1280, 1997.

HU, W.; MURPHY, M. R.; CONSTABLE, P. D. et al. Dietary cation-anion difference and dietary protein effects on performance and acid-base status of dairy cows in early lactation. Journal of Dairy Science, v.90, n.7, p.3355-3366, 2007.

HUTJENS, M.; AALSETH, E. Caring for transition cows. Fort Atkinson: W.D. Hoards \& Sons Company, 2005. 64p.

JARDON, P. Using urine $\mathrm{pH}$ to monitor anionic salt programs. Compendium on Continuing Education for the Practicing Veterinarian. v.17, p.860, 1995.

LEITE, L. C.; ANDRIGUETTO, J. L.; PAULA, M. C. et al. Diferentes balanços catiônicosaniônicos da dieta de vacas da raça holandesa. Revista Brasileira de Zootecnia, v.32, n.5, p.1259-1265, 2003.

\section{NATIONAL RESEARCH COUNCIL. Nutrient}

Requirements of Dairy Cattle. 7. ed.

Washington: National Academy Press. 2001. $380 \mathrm{p}$.

OETZEL, G. R. Monitoring and testing dairy herds for metabolic diseases. The Veterinary Clinics of North America: Food Animal Practice, v.20, n.3, p.651-674, 2004.

OVERTON, T. R.; NYDAM, D. V.; WELCOME, $F$. et al. Transition cow guidelines within the first 60 days in milk as a percentage of calvings. New York State Cattle Health Assurance Program, 2002. Disponível em:

<http://www.nyschap.vet.cornell.edu/factsheet/fa cts.asp>. Acesso em: 14/12/2009.
RAMOS-NIEVES, J. M.; THERING, B. J.; WALDRON, M. R. et al. Effects of anion supplementation to low-potassium prepartum diets on macromineral status and performance of periparturient dairy cows. Journal of Dairy Science, v.92, n.11, p.5677-5691, 2009.

ROCHE, J. R.; DALLEY, D. E.; MOATE, P. et al. Dietary cation-anion difference and the health and production of pasture-fed dairy cows. 1 . Dairy cows in early lactation. Journal of Dairy Science, v.86, n.3, p.970-978, 2003.

ROCHE, J. R.; DALLEY, D. E.; O’MARA, F. P. Effect of a metabolically created systemic acidosis on calcium homeostasis and the diurnal variation in urine $\mathrm{pH}$ in the non-lactating pregnant dairy cow. Journal of Dairy Research, v.74, n.1, p.34-39, 2007.

SAS Institute. SAS User's Guide. Version 9.1. Ed. SAS Institute Inc., Cary, NC, 2002.

SEIFI, H. A.; MOHRI, M.; KALAMATI ZADEH, J. Use of pre-partum urine $\mathrm{pH}$ to predict the risk of milk fever in dairy cows. The Veterinary Journal, v.167, n.3, p.281-285, 2004.

WU, W. X.; LIU, J. X.; XU, G. Z. et al. Calcium homeostasis, acid-base balance, and health status in periparturient Holstein cows fed diets with low cation-anion difference. Livestock Science, v.117, n.1, p.7-14, 2008. 